\title{
Research on the Object-oriented Classification Method of SAR Marine Oil Spill Image based on eCognition Software
}

\author{
$\mathrm{Li} \mathrm{Na}^{1, \mathrm{a}, *}$, Nie Xiujun ${ }^{2, \mathrm{~b}}$, Cui Miaomiao,c \\ ${ }^{1}$ Architectural Engineering Department, Binzhou Polytechnic, Yellow River Road 12, Binzhou, China \\ ${ }^{2}$ Department of Oceanography, Binzhou Polytechnic, Yellow River Road 12, Binzhou, China \\ a 411245815@qq.com, b 443183538@qq.com, c 110384954@qq.com \\ *corresponding author
}

Keywords: marine oil spill, SAR image, object-oriented classification method.

\begin{abstract}
Because oil spill accidents occur frequently in bad weather and environment, SAR images with all-weather and all-time characteristics have become one of the main remote sensing methods to detect oil spill. In this paper, based on the data of SAR marine oil spill image and eCognition software, the object-oriented classification method is studied and applied. The purpose of this paper is to serve the remote sensing monitoring of marine oil spills, provide a certain reference for the identification of offshore oil film, and provide a certain basis for the monitoring and control of offshore oil pollution.
\end{abstract}

\section{Introduction}

With the accelerated pace of exploitation and utilization of marine oil resources, marine pollution has also accompanied. According to statistics, oil pollution ranks first among all kinds of marine pollution. Marine oil spill accidents have high frequency, wide distribution and deep damage, which seriously hinder the sustainable development of the marine environment. Because oil spill accidents occur frequently in bad weather and environment, SAR images with all-weather and all-time characteristics have become one of the main remote sensing methods to detect oil spill. The data used in this paper is the ASAR data of ENVISAT-1 satellite, which recorded the oil spill accident of Prestige in 2002.

Traditional remote sensing image classification methods usually only take a single pixel as the classification object, which is the classification on the pixel level, and the information used for classification is only spectral information. Compared with the traditional remote sensing image classification method, the object of object-oriented method is the homogeneous region, which is the patches formed after segmentation on the remote sensing image. In addition, the object area contains a lot of information. For oil spill SAR images, in addition to gray information, there will be texture, shape, location and so on. With the information, the classification results will be more accurate and reasonable. In this paper, the SAR image of oil spill is taken as the research data, and the object-oriented classification method is studied and applied based on eCognition software. The purpose is to serve the remote sensing monitoring of oil spill, provide a certain reference for the identification of oil film on the sea, and provide a certain basis for the monitoring and control of marine oil pollution.

\section{Multiscale segmentation}

According to some same attributes, the space is divided into several non-overlapping regions, 
and then the targets of interest is extracted. This process is image segmentation. Image segmentation is based on regional similarity and discontinuity. The segmentation algorithm provided by eCognition software is multi-scale segmentation, four fork tree segmentation and chessboard segmentation. Multiscale segmentation utilizes different segmentation scales to construct mesh topology through multiple segmentation. Multiscale segmentation adopts the bottom-up growth method, merging from the bottom pixel. The specific process is: starting from the underlying pixels, the pixels with similar features are merged into small regions, and then merged into large regions based on the principle of minimum heterogeneity. Each merge must calculate the heterogeneity of the two regions, and then compared with the user-specified scale. If it is larger than the user-specified scale, it does not merge, otherwise it merges. The next merger will be based on the previous merger until the merger is completed or the regional heterogeneity is larger than user-specified scale.

In order to ensure classification accuracy, it is a crucial step to choose an optimal scale for segmentation. Under the optimal scale, we should be able to recognize the accurate outline of the object, but not create too many fine polygons. Therefore, in practice, the scaling parameter selection of eCognition should follow the following principles: (1) Under the condition of satisfying the necessary precision, the maximum segmentation scale should be used as far as possible; (2) Color standard should be adopted as far as possible under the condition of satisfying the necessary shape requirements; (3) Large-scale objects correspond to large-scale, small-scale objects correspond to small-scale; (4) Full-scale objects set large compactness weight; Smooth objects set large smoothness. The following is the segmented image:

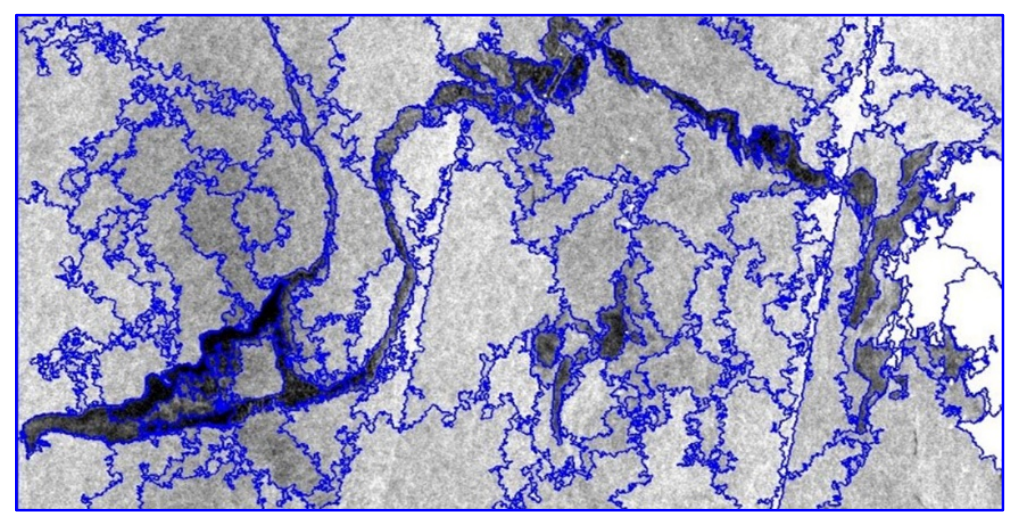

Figure 1 Multiscale segmented image.

\section{Selection of classification features}

Feature is the description of attributes of classification objects, and is also the main basis for image recognition and classification. The essence of remote sensing image classification is to classify objects with similar features into one category. Therefore, the selection of classification features and the establishment of feature space are crucial steps. In eCognition software, the feature information of the object is very rich. According to the characteristics of the object itself, we can choose the appropriate feature. In order to select the feature intuitively and correctly, eCognition software also provides the function of viewing all kinds of feature image in Feature View. Therefore, through repeated debugging comparison, we can determine the feature space involved in classification. 


\section{Image classification}

The classification methods commonly used in eCognition software include threshold classification, fuzzy classification and supervised classification.

\subsection{Threshold classification}

Threshold is artificially set and it is used to decide whether the object area matches the relevant conditions. If only one feature can be used to distinguish between different categories, the simplest threshold segmentation is usually chosen in this case. In the oil spill SAR image, the gray feature is undoubtedly the most obvious of all the features for oil film region discrimination. If we do not consider the existence of oil film-like substances, and only divide the image into oil spill targets and background areas, then the single threshold method is sufficient. Based on this, this paper tests the classification of single threshold method. A threshold is used to classify the image into two parts: the target area and the background area. The image after classification is shown in Figure 2 below.

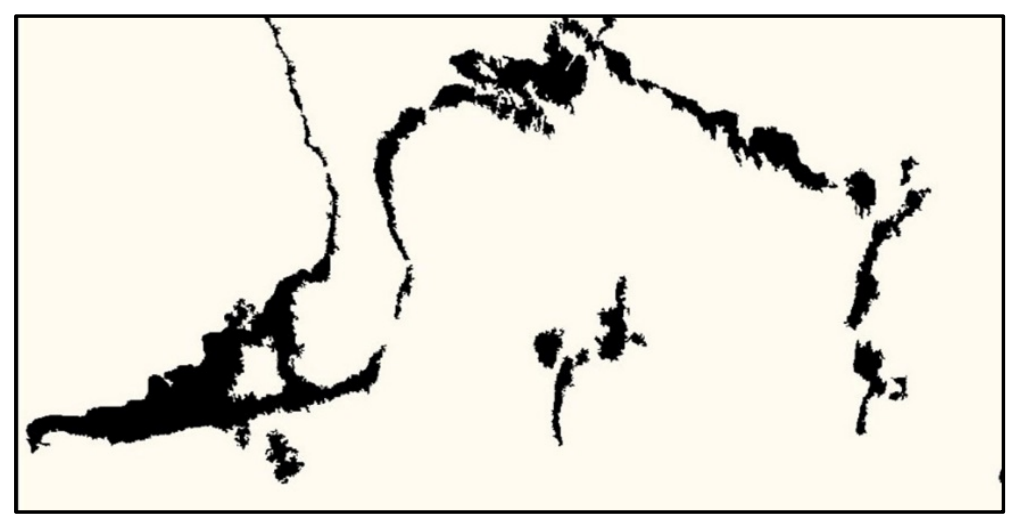

Figure 2 Image after single threshold classification.

It is difficult to distinguish oil film and other substances similar to oil film by single threshold method. If we want to distinguish four kinds of objects, we need three thresholds. The class description function in eCognition rule set mode can realize multi threshold segmentation. For this oil spill SAR image, the foreground area is classified as oil film and oil film, and the background area is classified as land and sea water. The threshold effect can be seen in the software through experimental modulation, which is very intuitive. The result of multi threshold classification is shown in Figure 3 below.

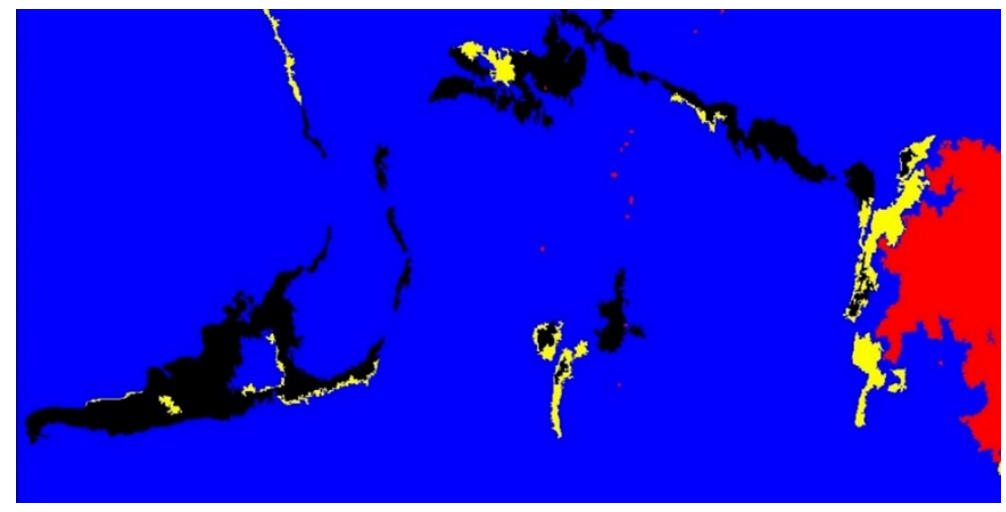

Figure 3 Image after multi-threshold classification.

From the classification results, it can be seen that although land and sea water can be effectively distinguished, this method is not very ideal for oil film and similar oil film discrimination. The reason is that it is difficult to distinguish oil film from similar oil film only by a single threshold set 
by a feature. This method is not very suitable for the classification of oil spill images.

\subsection{Fuzzy classification}

Fuzzy classification is also based on the classification characteristics of objects to classify, it is by calculating the membership of the object to determine which category it should belong to. Unlike previous algorithms, membership function is no longer a simple decision whether yes or no, but provides a range. After the feature evaluation is completed, the membership function will return a number between $0-1$, which is called membership degree. Each characteristic value of each class corresponds to a membership degree. These values can be combined by logical operations to perform class assignment calculations. Therefore, membership functions can combine different dimensions and ranges. The membership function can also edit and adjust each feature to make the relationship between the classification eigenvalue and membership clear.

According to the threshold classification, if the oil film and similar oil film are defined as foregrounds, sea water and land are defined as backgrounds, the foreground and background can be well distinguished by a single threshold, but the threshold method is difficult to distinguish between oil film and similar oil film. Therefore, membership functions need to be introduced. By choosing the membership function, we find that the effect is not good, so we need to define the membership function by ourselves. Through repeated tests, it is found that the distinction is different between oil film and similar oil film in 0-85 and 85-100. Therefore, two sets of membership functions are defined between $0-100$. The inflection points in the function are the distinguishing points of the characteristic values of different oil film and similar oil film regions obtained by software test. Figure 4 below is the image after fuzzy classification.

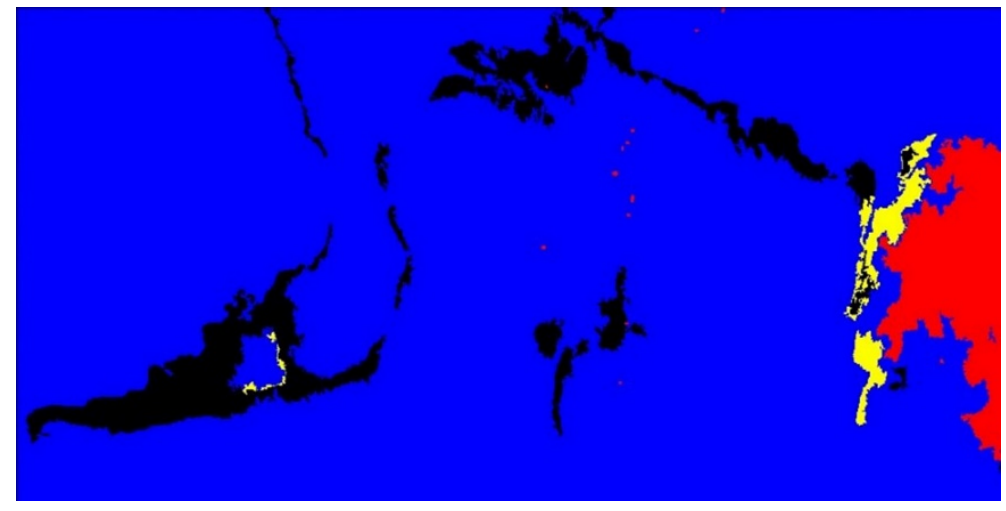

Figure 4 Image after fuzzy classification.

\subsection{Supervised classification}

In the supervised classification provided by eCognition software, the nearest neighbor classifier is often used because of its simple principle and high efficiency. The nearest neighbor classification is also based on feature space and object selection. After the sample object of each class is declared, the object area to be classified is divided into the class represented by the nearest sample. There are two nearest neighbor expressions in eCognition software, that is, nearest neighbor and standard nearest neighbor. The difference between them is that the nearest neighbor feature space can define each individual class, while the standard nearest neighbor feature space can be applied to the entire project or to all categories of the expression. Nearest neighbor classification is similar to traditional supervised classification, which is based on training samples to select object characteristics. It can generate multidimensional membership functions. Compared with pixel based supervised classification, there are fewer object oriented samples. Its principle is to calculate the distance from the classification object to the feature center, and classify the object into the nearest category. 
In eCognition environment, feature space selection and sample selection are needed when nearest neighbor classification is applied to images. The properties of each object can be viewed through the Feature View in eCognition to assist in defining the feature space of the nearest neighbor expression. When samples are selected, a small number of samples are selected for each class to conduct experiments. After multiple sample selection and experimental classification, a nearest neighbor classifier suitable for this image can be obtained. It should be noted that after each experiment, the wrong or missed points should be selected as samples, and continue to participate in classification until the results are satisfactory. Class editing dialog box of eCognition software can show the distribution of each sample and the specific eigenvalues. Through this dialog box, it is easy to compare and modify samples. The results of the nearest neighbor method for supervised classification are shown in Figure 5 below:

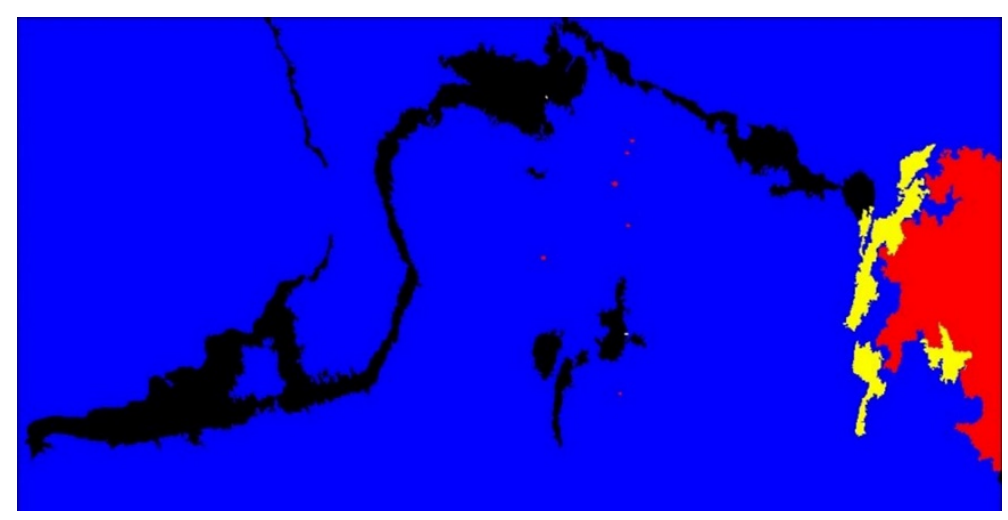

Figure 5 Image after nearest neighbor classification.

\section{Summary}

In this paper, the SAR image of marine oil spill is taken as the research object. Based on eCognition software, the image is processed by object-oriented classification method, and the oil spill area is effectively identified. For the experimental results in this paper, the classification effect of fuzzy classification and supervisory classification is better than threshold classification. Through the application and comparison of different classification methods, this paper aims at serving the remote sensing monitoring of marine oil spill, providing a certain reference for marine oil film identification, and providing a certain basis for the monitoring and control of marine oil pollution.

\section{References}

[1] Li Ying, Li Guannan, Cui Gan. Research Progress of Oil Spill Detection based on Spaceborne SAR[J].Ocean Circular, 2017 (6): 241-248.

[2] Zhou Hui, Chen Peng. SAR Oil Spill Image Recognition Method based on RBF Network Model[J].Journal of Dalian Maritime University, 2018 (5): 113-117.

[3] Wang Zhiyong, Wang Shishuai, Wang Shichao. Optimal Segmentation Methods for Different Oil Film Features in SAR Images [J].Surveying and Mapping Engineering, 2017 (1): 1-7.

[4] Su Tengfei, Li Yongxiang, Li Hongyu. SAR Oil Spill Detection Algorithm based on Object-oriented and Fuzzy Logic[J].Journal of Oceanography, 2016 (1): 69-77.

[5] Wu Tengteng, Zhu Hao, Fan Yanguo, Mao Guangshui. Application of Minimum Fuzzy Entropy Algorithm in SAR Image Oil Spill Detection[J]. Remote Sensing Information, 2015(4): 89-93. 